\title{
Sums of orbits of integral matrices
}

\author{
Cristina Caldeira, João Filipe Queiró \\ CMUC, Department of Mathematics, University of Coimbra, \\ 3001-501 Coimbra, Portugal
}

\begin{abstract}
For square matrices $A$ and $B$ over an elementary divisor domain, we study the possible invariant factors of $A+B$ in terms of the invariant factors of $A$ and $B$. In particular, we find the exact range of $\operatorname{det}(A+B)$ in that situation.
\end{abstract}

Keywords: Invariant factors, elementary divisor domains, determinants

AMS Subject Classifications: 15A15; 15B33; 15B36

\section{Introduction}

In this note we work with $n \times n$ matrices over principal ideal domains or, more generally, elementary divisor domains $R$, defined by the condition that every matrix is equivalent to its Smith normal form (for a recent treatment of these rings, see [3]). We shall be concerned with the action on $R^{n \times n}$ defined by $A \mapsto$ $U A V$, where $U, V$ are invertible matrices over $R$. The orbits for this action are characterized by $n$-tuples of elements forming divisibility chains, the invariant factors.

For such an $n$-tuple $a$, we denote by $\mathcal{O}_{a}$ the corresponding orbit. A problem that attracted attention for a long time was the description of $\mathcal{O}_{a} \mathcal{O}_{b}$ for given $a$ and $b$. An answer in the principal ideal domain case was provided by Klein in 1968 [5], working with modules and localizing over a prime, in terms of LittlewoodRichardson sequences. An explicit solution involving divisibility relations was found much later (see e.g. [9] for the relation between the module and matrix problems and [4] for an account of the solution). Recently, the same list of divisibility relations was shown to be necessary for the product problem in the larger class of elementary divisor domains [1]. In all this work, the invariant factor product problem was seen to be a deep question, with relations to various important areas of mathematics.

In the 1980s, the analogous additive problem, that of describing $\mathcal{O}_{a}+\mathcal{O}_{b}$ for given chains of invariant factors $a$ and $b$, also received some attention, in particular by R. C. Thompson, one of the main protagonists in the study of the product 
question. After some contributions (see below), with no special structural framework, the sum problem remains open. So, given three divisibility chains with the same length, it is not known when the third chain is the invariant factor sequence of a sum of two matrices having the elements of the other two chains as invariant factors.

Here we make some progress on this problem, in two ways. First, we shall be concerned with a natural function of the third sequence, namely the product of its elements. In other words, we aim at describing the possible values of the determinant of the sum of two matrices with given invariant factors. We present a complete solution to this problem for matrices over elementary divisor domains. Second, we relate this to previous work and present a new conjecture for the solution of the sum problem.

For matrices of the form $\lambda I-A$ over the polynomial ring $\mathbb{K}[\lambda]$, where $\mathbb{K}$ is a field, the question is known as the additive Deligne-Simpson problem and it is considered in [2], with deep connections to representation theory.

\section{Invariant factors of sums}

For completeness, we briefly describe what is known about invariant factors of sums of matrices.

Our notation is standard. Let $n \geq 2$. Given $n \times n$ matrices $A$ and $B$ over an elementary divisor domain $R$, we denote their invariant factors by $a_{1}|\cdots| a_{n}$ and $b_{1}|\cdots| b_{n}$, respectively. As is well-known, we have

$$
a_{k}=\frac{d_{k}(A)}{d_{k-1}(A)}, k=1, \ldots, \operatorname{rank}(A), a_{k}=0 \text { for } k>\operatorname{rank}(A),
$$

where, for each $k, d_{k}(A)$ is the gcd of all $k \times k$ minors of $A, d_{0}:=1$. This means that the invariant factors of a matrix are well defined apart from units in the ring. Also, we clearly have $d_{k}(A)=a_{1} \cdots a_{k}$, apart from unit factors, for all $k$.

In [10], R. C. Thompson proved that, if $c_{1}|\cdots| c_{n}$ are the invariant factors of $A+B$, then

$$
\operatorname{gcd}\left\{a_{i}, b_{j}\right\} \mid c_{i+j-1}
$$

for all indices $i, j$ such that $i+j-1 \leq n$. His proof uses localization at a prime, requiring $R$ to be a principal ideal domain, but the result is valid for matrices over an elementary divisor domain (see the argument in [7]).

Write $A+B=C$. From the equalities $B=-A+C$ and $A=-B+C$, we see that we have not one but three families of relations

$$
\operatorname{gcd}\left\{a_{i}, b_{j}\right\}\left|c_{i+j-1} \quad, \quad \operatorname{gcd}\left\{a_{i}, c_{j}\right\}\right| b_{i+j-1} \quad \text { and } \operatorname{gcd}\left\{b_{i}, c_{j}\right\} \mid a_{i+j-1} .
$$


Also, trivially, taking determinants (with invariant factors chosen so that their product equals the determinant) we have

$$
\begin{gathered}
a_{1} \cdots a_{n} \equiv c_{1} \cdots c_{n}\left(\bmod b_{1}\right), \\
b_{1} \cdots b_{n} \equiv c_{1} \cdots c_{n}\left(\bmod a_{1}\right), \\
a_{1} \cdots a_{n} \equiv(-1)^{n} b_{1} \cdots b_{n}\left(\bmod c_{1}\right) .
\end{gathered}
$$

In [11], Thompson conjectured that, for $n \geq 2$, the six conditions (2) and (3) are the complete solution for the invariant factor sum problem, i.e. that, if they hold (with $a_{1}, b_{1}, c_{1}$ relatively prime, which entails no loss of generality), then matrices $A \in \mathcal{O}_{a}, B \in \mathcal{O}_{b}$ exist such that $A+B \in \mathcal{O}_{c}$.

In [8], E. Marques de Sá showed that Thompson's conjecture is false in the general case, by finding additional necessary conditions (see the final section).

\section{Determinants of sums}

A source of inspiration when looking for invariant factor relations is the corresponding situation for singular values of complex matrices. The analogy is wellknown (see e.g. [7], [4]). So it is of interest that, in [6], the following inequality was proved:

$$
\prod_{i=1}^{n}\left(\alpha_{i}+\beta_{n-i+1}\right) \geq \operatorname{det}(A+B)
$$

where $A$ and $B$ are $n \times n$ complex matrices with singular values $\alpha_{1} \geq \cdots \geq \alpha_{n}$ and $\beta_{1} \geq \cdots \geq \beta_{n}$.

In keeping with the analogy, we should replace sum by gcd on the left-side, as in relation (1) above (without that, the result is easily seen to be false). Indeed, we have:

Theorem 1. Let $A$ and $B$ be $n \times n$ matrices over $R$ with invariant factors $a_{1}|\cdots| a_{n}$ and $b_{1}|\cdots| b_{n}$. Then

$$
\prod_{i=1}^{n} \operatorname{gcd}\left\{a_{i}, b_{n-i+1}\right\} \mid \operatorname{det}(A+B) \text {. }
$$

Proof. We have the well-known formula for determinants of sums:

$$
\operatorname{det}(A+B)=\sum_{k=0}^{n} \sum_{\mu, \nu \in Q_{k, n}}(-1)^{\sum \mu+\sum \nu} \operatorname{det} A[\mu \mid \nu] \cdot \operatorname{det} B\left[\mu^{\prime} \mid \nu^{\prime}\right]
$$


where $Q_{k, n}$ is the set of strictly increasing sequences with $k$ elements taken from $\{1,2, \ldots, n\}, A[\mu \mid \nu]$ is the submatrix of $A$ with rows and columns indexed by $\mu$ and $\nu$, and $\mu^{\prime}, \nu^{\prime}$ are the complementary sequences to $\mu, \nu$.

Apart from the sign, each summand is the product of a $k \times k$ minor of $A$, which is a multiple of $a_{1} \cdots a_{k}$, by a $(n-k) \times(n-k)$ minor of $B$, which is a multiple of $b_{1} \cdots b_{n-k}$. The whole sum clearly must be a multiple of $\prod_{i=1}^{n} \operatorname{gcd}\left\{a_{i}, b_{n-i+1}\right\}$.

Condition (4) is simple and elegant but it is not the complete solution to the determinant question. An example showing this is the following:

Example. Consider $R=\mathbb{Z}$ and $n=2$. For any matrices $A$ and $B$ with invariant factors $2 \mid 4$ and $3 \mid 6, \operatorname{det}(A+B)$ is never a multiple of 6 . So, not every multiple of $\operatorname{gcd}\{2,6\} \operatorname{gcd}\{4,3\}=2$ is attainable as the determinant of the sum of two matrices with the prescribed invariant factors.

We must take formula (5) a bit further. In the following statement, we choose the invariant factors of a matrix so that their product equals the determinant of the matrix.

Theorem 2. Let $A$ and $B$ be $n \times n$ matrices over $R$ with invariant factors $a_{1}$ $\cdots \mid a_{n}$ and $b_{1}|\cdots| b_{n}$. Put $\delta=\operatorname{gcd}\left\{a_{1} \cdots a_{k} b_{1} \cdots b_{n-k}: k=1, \ldots, n-1\right\}$. Then

$$
\operatorname{det}(A+B) \equiv a_{1} \cdots a_{n}+b_{1} \cdots b_{n}(\bmod \delta) \text {. }
$$

Proof. This is immediate from formula (5). For $k \in\{1, \ldots, n-1\}$ and $\mu, \nu \in Q_{k, n}$, we have $\operatorname{det} A[\mu \mid \nu] \equiv 0\left(\bmod a_{1} \cdots a_{k}\right)$ and $\operatorname{det} B\left[\mu^{\prime} \mid \nu^{\prime}\right] \equiv 0\left(\bmod b_{1} \cdots b_{n-k}\right)$, so

$$
\operatorname{det} A[\mu \mid \nu] . \operatorname{det} B\left[\mu^{\prime} \mid \nu^{\prime}\right] \equiv 0\left(\bmod a_{1} \cdots a_{k} b_{1} \cdots b_{n-k}\right) .
$$

Summing for all $k$, we get from (5) that

$$
\operatorname{det}(A+B) \equiv \operatorname{det}(A)+\operatorname{det}(B)(\bmod \delta)
$$

which is the same as

$$
\operatorname{det}(A+B) \equiv a_{1} \cdots a_{n}+b_{1} \cdots b_{n}(\bmod \delta),
$$

as required.

We proceed to show that condition (6) is also sufficient. 
First, a technical lemma.

Lemma 1. For $n \geq 2$ and $\alpha_{1}, \ldots, \alpha_{n-1}, \beta_{1}, \ldots, \beta_{n-1}, \gamma_{1}, \ldots, \gamma_{n} \in R$, we have

$$
\operatorname{det}\left[\begin{array}{cccccc}
\alpha_{1} & & & & & \gamma_{1} \\
\beta_{1} & \alpha_{2} & & 0 & & \gamma_{2} \\
& \beta_{2} & \alpha_{3} & & & \gamma_{3} \\
& & \ddots & \ddots & & \vdots \\
& 0 & & \beta_{n-2} & \alpha_{n-1} & \gamma_{n-1} \\
& & & & \beta_{n-1} & \gamma_{n}
\end{array}\right]=\sum_{i=1}^{n}(-1)^{n+i} \gamma_{i} \prod_{j=1}^{i-1} \alpha_{j} \prod_{j=i}^{n-1} \beta_{j}
$$

Proof. The case $n=2$ is trivial. For $n \geq 3$, we get, using Laplace's Theorem,

$$
\begin{aligned}
& \operatorname{det}\left[\begin{array}{ccccc}
\alpha_{1} & & & & \gamma_{1} \\
\beta_{1} & \alpha_{2} & & 0 & \gamma_{2} \\
& \ddots & \ddots & & \vdots \\
& & \beta_{n-2} & \alpha_{n-1} & \gamma_{n-1} \\
& 0 & & \beta_{n-1} & \gamma_{n}
\end{array}\right]= \\
& -\beta_{n-1} \operatorname{det}\left[\begin{array}{ccccc}
\alpha_{1} & & & & \gamma_{1} \\
\beta_{1} & \alpha_{2} & 0 & & \gamma_{2} \\
& \ddots & \ddots & & \vdots \\
& & \beta_{n-3} & \alpha_{n-2} & \gamma_{n-2} \\
& 0 & & \beta_{n-2} & \gamma_{n-1}
\end{array}\right]+\gamma_{n} \operatorname{det}\left[\begin{array}{ccccc}
\alpha_{1} & & & & \\
\beta_{1} & \alpha_{2} & & 0 & \\
& \beta_{2} & \alpha_{3} & & \\
& & \ddots & \ddots & \\
& 0 & & \beta_{n-2} & \alpha_{n-1}
\end{array}\right]
\end{aligned}
$$

and the result follows by induction on $n$.

Theorem 3. Take sequences $a_{1}|\cdots| a_{n}$ and $b_{1}|\cdots| b_{n}$ of elements of $R$. Define $\delta$ as in Theorem 2. Suppose that an element $x \in R$ satisfies $x \equiv$ $a_{1} \cdots a_{n}+b_{1} \cdots b_{n}(\bmod \delta)$. Then there exist $n \times n$ matrices $A$ and $B$ with invariant factors $a_{1}|\cdots| a_{n}$ and $b_{1}|\cdots| b_{n}$ such that $\operatorname{det}(A+B)=x$.

Proof. For $n=2$, let $x=a_{1} a_{2}+b_{1} b_{2}+q a_{1} b_{1}$. Then the matrices $A=\left[\begin{array}{cc}a_{1} & 0 \\ q a_{1} & a_{2}\end{array}\right]$ and $B=\left[\begin{array}{cc}0 & -b_{1} \\ b_{2} & 0\end{array}\right]$ satisfy the required. 
Suppose $n \geq 3$ and let $x \in R$ be such that $x \equiv a_{1} \cdots a_{n}+b_{1} \cdots b_{n}(\bmod \delta)$. Since every elementary divisor domain is also a Bézout domain, there exist $q_{1}, \ldots, q_{n-1} \in$ $R$ such that $x=a_{1} \cdots a_{n}+b_{1} \cdots b_{n}+\sum_{k=1}^{n-1} q_{k} a_{1} \cdots a_{k} b_{1} \cdots b_{n-k}$.

Consider the matrices $A=\left[\begin{array}{cc}a_{1} & 0 \\ q_{1} a_{1} & a_{2}\end{array}\right] \oplus \operatorname{diag}\left(a_{3}, \ldots, a_{n}\right)$ and

$$
B=\left[\begin{array}{c|c}
0 & (-1)^{n-1} b_{1} \\
\hline \operatorname{diag}\left(b_{n}, b_{n-1}, \ldots, b_{2}\right) & (-1)^{n-3} q_{2} b_{1} \\
\vdots \\
-q_{n-2} b_{1} \\
q_{n-1} b_{1}
\end{array}\right] \text {. }
$$

By means of elementary row and column operations we see that $A$ and $B$ are equivalent to $\operatorname{diag}\left(a_{1}, a_{2}, \ldots, a_{n}\right)$ and $\operatorname{diag}\left(b_{1}, b_{2}, \ldots, b_{n}\right)$, respectively. Hence $a_{1}|\cdots| a_{n}$ and $b_{1}|\cdots| b_{n}$ are the invariant factors of $A$ and $B$.

From the previous Lemma we have

$$
\begin{aligned}
\operatorname{det}(A+B)= & \operatorname{det}\left[\begin{array}{ccccc}
a_{1} & & & & (-1)^{n-1} b_{1} \\
q_{1} a_{1}+b_{n} & a_{2} & & & \\
& b_{n-1} & a_{3} & & 0 \\
& & \ddots & \ddots & (-1)^{n-3} q_{2} b_{1} \\
& & & & \vdots \\
& & b_{3} & a_{n-1} & -q_{n-2} b_{1} \\
& & & b_{2} & a_{n}+q_{n-1} b_{1}
\end{array}\right] \\
= & b_{1}\left(q_{1} a_{1}+b_{n}\right) b_{2} \cdots b_{n-1}+\sum_{i=3}^{n-1} q_{i-1} a_{1} \cdots a_{i-1} b_{1} \cdots b_{n-i+1}+ \\
& \left(a_{n}+q_{n-1} b_{1}\right) a_{1} \cdots a_{n-1} \\
= & x .
\end{aligned}
$$

So condition (6) is necessary and sufficient for the existence of two matrices with the given invariant factors and such that the determinant of their sum has the prescribed value. 


\section{Further notes on the sum problem}

At first sight, Thompson's conjecture is quite a bold one, since it simply joins, as possible sufficient conditions for the sum problem, the divisibility relations (2) to the trivial congruences (3). But, in fact, the relations (2) are highly restrictive on the three $n$-tuples of invariant factors, so the conjecture was not unnatural. Sá showed it is not true, but the conditions he added in [8] are just modifications of (3).

Let's illustrate how restrictive conditions (2) are. We might think of using formula (5) as in Theorem 2 to obtain some additional necessary conditions. Let $m \in\{1,2, \ldots, n\}$. For every $\xi, \zeta \in Q_{m, n}$ we have

$$
\operatorname{det}(A+B)[\xi \mid \zeta]=\sum_{k=0}^{m} \sum_{\mu, \nu \in Q_{k, m}}(-1)^{\sum \mu+\sum \nu} \operatorname{det} A[\xi \mid \zeta][\mu \mid \nu] \cdot \operatorname{det} B[\xi \mid \zeta]\left[\mu^{\prime} \mid \nu^{\prime}\right],
$$

and so every $m \times m$ minor of $A+B$ is a multiple of

$\varepsilon_{m}=\operatorname{gcd}\left\{a_{1} \cdots a_{m}, a_{1} \cdots a_{m-1} b_{1}, a_{1} \cdots a_{m-2} b_{1} b_{2}, \ldots, a_{1} b_{1} \cdots b_{m-1}, b_{1} \cdots b_{m}\right\}$.

Hence, if $c_{1}|\cdots| c_{n}$ are the invariant factors of $A+B$, we have

$$
c_{1} c_{2} \cdots c_{m} \equiv 0\left(\bmod \varepsilon_{m}\right), \quad m=1, \ldots, n .
$$

But these $n$ congruences actually are not new necessary conditions, since they are a consequence of $(2)$ :

Theorem 4. If $a_{1}|\cdots| a_{n}, b_{1}|\cdots| b_{n}$ and $c_{1}|\cdots| c_{n}$ are sequences of elements in $R$ that satisfy $(2)$ then $c_{1} c_{2} \cdots c_{m} \equiv 0\left(\bmod \varepsilon_{m}\right)$, for all $m=1, \ldots, n$.

Proof. For each $m \in\{2, \ldots, n\}$,

$$
\begin{aligned}
& \varepsilon_{m}=\operatorname{gcd}\left\{a_{1} \cdots a_{m-1} \operatorname{gcd}\left\{a_{m}, b_{1}\right\}, a_{1} \cdots a_{m-2} b_{1} \operatorname{gcd}\left\{a_{m-1}, b_{2}\right\}, \ldots,\right. \\
& \left.a_{1} b_{1} \cdots b_{m-2} \operatorname{gcd}\left\{a_{2}, b_{m-1}\right\}, b_{1} \cdots b_{m-1} \operatorname{gcd}\left\{a_{1}, b_{m}\right\}\right\} .
\end{aligned}
$$

From (2) we have that $\operatorname{gcd}\left\{a_{m-k-1}, b_{k}\right\} \mid c_{m}$, for $k=1, \ldots, m$ and so $\varepsilon_{m} \mid$ $\varepsilon_{m-1} c_{m}$. Since $\varepsilon_{1}=\operatorname{gcd}\left\{a_{1}, b_{1}\right\} \mid c_{1}$ it follows that $\varepsilon_{m} \mid c_{1} c_{2} \cdots c_{m}$.

Given Theorem 2, we can add condition (6) to the list of necessary conditions on invariant factors of sums of matrices with given invariant factors. It is natural to ask how it relates to Thompson's conjecture and Sá's later work. To do this 
we present all the known necessary conditions, renaming them for convenience. For symmetry purposes we follow [8] and consider matrices $A, B$ and $C$ such that $A+B+C=0$. So, if $A, B$ and $C$ are $n \times n$ matrices with invariant factors $a_{1}\left|a_{2}\right| \cdots\left|a_{n}, \quad b_{1}\right| b_{2}|\cdots| b_{n}$ and $c_{1}\left|c_{2}\right| \cdots \mid c_{n}$, chosen so that the determinant of each matrix is equal to the product of its invariant factors, and $A+B+C=0$, then the following conditions hold:

(I) $\quad\left\{\begin{array}{l}\operatorname{gcd}\left\{a_{i}, b_{j}\right\} \mid c_{i+j-1} \\ \operatorname{gcd}\left\{a_{i}, c_{j}\right\} \mid b_{i+j-1} \quad, \quad 1 \leq i, j \leq n, \quad i+j-1 \leq n ; \\ \operatorname{gcd}\left\{c_{i}, b_{j}\right\} \mid a_{i+j-1}\end{array}\right.$

(II) $\left\{\begin{array}{l}\prod_{i=1}^{n} a_{i} \equiv(-1)^{n} \prod_{i=1}^{n} b_{i}\left(\bmod c_{1} \delta_{1}^{n-1}\right) \\ \prod_{i=1}^{n} b_{i} \equiv(-1)^{n} \prod_{i=1}^{n} c_{i}\left(\bmod a_{1} \delta_{1}^{n-1}\right) \\ \prod_{i=1}^{n} c_{i} \equiv(-1)^{n} \prod_{i=1}^{n} a_{i}\left(\bmod b_{1} \delta_{1}^{n-1}\right)\end{array}\right.$

where $\delta_{1}=\operatorname{gcd}\left\{a_{1}, b_{1}, c_{1}\right\}$;

$$
\left\{\begin{array}{l}
\prod_{i=1}^{n} a_{i} \equiv(-1)^{n} \prod_{i=1}^{n} b_{i}(\bmod \gamma) \\
\prod_{i=1}^{n} b_{i} \equiv(-1)^{n} \prod_{i=1}^{n} c_{i}(\bmod \alpha) \\
\prod_{i=1}^{n} c_{i} \equiv(-1)^{n} \prod_{i=1}^{n} a_{i}(\bmod \beta)
\end{array}\right.
$$

where

$$
\alpha=a_{1} \prod_{i=1}^{n-1} \operatorname{gcd}\left\{a_{1}, b_{i}\right\}, \quad \beta=b_{1} \prod_{i=1}^{n-1} \operatorname{gcd}\left\{b_{1}, c_{i}\right\}, \quad \gamma=c_{1} \prod_{i=1}^{n-1} \operatorname{gcd}\left\{c_{1}, a_{i}\right\} ;
$$

and 
(IV)

$$
\left\{\begin{aligned}
(-1)^{n} \prod_{i=1}^{n} c_{i} & \equiv \prod_{i=1}^{n} a_{i}+\prod_{i=1}^{n} b_{i}(\bmod \delta) \\
(-1)^{n} \prod_{i=1}^{n} a_{i} & \equiv \prod_{i=1}^{n} b_{i}+\prod_{i=1}^{n} c_{i}(\bmod \theta) \\
(-1)^{n} \prod_{i=1}^{n} b_{i} & \equiv \prod_{i=1}^{n} a_{i}+\prod_{i=1}^{n} c_{i}(\bmod \eta)
\end{aligned}\right.
$$

where

$$
\begin{aligned}
& \delta=\operatorname{gcd}\left\{a_{1} \cdots a_{k} b_{1} \cdots b_{n-k}: k=1, \ldots, n-1\right\} \\
& \theta=\operatorname{gcd}\left\{c_{1} \cdots c_{k} b_{1} \cdots b_{n-k}: k=1, \ldots, n-1\right\} \\
& \eta=\operatorname{gcd}\left\{a_{1} \cdots a_{k} c_{1} \cdots c_{n-k}: k=1, \ldots, n-1\right\}
\end{aligned}
$$

(II) are Thompson's conditions from [11], in the version where we do not assume that $a_{1}, b_{1}, c_{1}$ are relatively prime. (III) are Sá's conditions from [8]. (IV) are our new necessary conditions obtained as in Theorem 2 from $C=-A-B, A=-B-C$ and $B=-A-C$.

Concerning conditions (I) to (IV) we have the following list of remarks.

Remark 1. Conditions (I) together with (III) imply (II) but conditions (I) together with (II) do not imply (III) [8].

Remark 2. Conditions (I) together with (II) do not imply (IV). Take, for instance, $n=3, \quad a: 1|2| 2, \quad b: 1|6| 30$ and $c: 8|16| 96$. This example also shows that the conditions in Theorem 4 do not imply (IV).

Remark 3. For $n$ odd, it is easy to show that (IV) are equivalent to

$$
\prod_{i=1}^{n} a_{i}+\prod_{i=1}^{n} b_{i}+\prod_{i=1}^{n} c_{i} \equiv 0(\bmod \operatorname{lcm}\{\delta, \theta, \eta\}),
$$

and (III) are equivalent to

$$
\prod_{i=1}^{n} a_{i}+\prod_{i=1}^{n} b_{i}+\prod_{i=1}^{n} c_{i} \equiv 0(\bmod \operatorname{lcm}\{\alpha, \beta, \gamma\})
$$

since $\alpha\left|\prod_{i=1}^{n} a_{i}, \beta\right| \prod_{i=1}^{n} b_{i}$ and $\gamma \mid \prod_{i=1}^{n} c_{i}$.

Remark 4. For $n=2$, the three sets of conditions $(\mathrm{I}) \wedge(\mathrm{II}),(\mathrm{I}) \wedge(\mathrm{III})$ and $(\mathrm{I}) \wedge(\mathrm{IV})$ are equivalent. This follows from elementary calculations. 
Next we prove that (IV) implies (III).

Lemma 2. If $a_{1}|\cdots| a_{n}, b_{1}|\cdots| b_{n}$ and $c_{1}|\cdots| c_{n}$ are sequences of elements in $R$ that satisfy (I) then

$$
\operatorname{lcm}\{\alpha, \beta\}|\delta, \quad \operatorname{lcm}\{\beta, \gamma\}| \theta \quad \text { and } \quad \operatorname{lcm}\{\alpha, \gamma\} \mid \eta .
$$

Proof. We will prove that $\alpha \mid \delta$ and $\beta \mid \delta$. The other relations are proved similarly. For each $k \in\{1, \ldots, n\}, a_{1} \cdots a_{k} b_{1} \cdots b_{n-k}$ is a multiple of $a_{1}^{k} b_{1} \cdots b_{n-k}$ and hence a multiple of $\alpha$. So $\alpha \mid \delta$.

Let $k \in\{1, \ldots, n\}$. From (I), $\operatorname{gcd}\left\{b_{1}, c_{i}\right\} \mid a_{i}$, for $i=1, \ldots, k$. Then

$$
\beta=b_{1} \prod_{i=1}^{n-1} \operatorname{gcd}\left\{b_{1}, c_{i}\right\}\left|a_{1} \cdots a_{k} b_{1}^{n-k}\right| a_{1} \cdots a_{k} b_{1} \cdots b_{n-k} .
$$

Therefore $\beta \mid \delta$.

This arguments shows that, even for sequences that do not satisfy (I), $\alpha \mid \delta$, $\beta \mid \theta$ and $\gamma \mid \eta$ will always hold.

Theorem 5. If $a_{1}|\cdots| a_{n}, b_{1}|\cdots| b_{n}$ and $c_{1}|\cdots| c_{n}$ are sequences of elements in $R$ that satisfy (IV) then they also satisfy (III).

Proof. The result follows promptly from $\alpha|\delta, \beta| \theta, \gamma|\eta, \alpha| \prod_{i=1}^{n} a_{i}$, $\beta \mid \prod_{i=1}^{n} b_{i}$ and $\gamma \mid \prod_{i=1}^{n} c_{i}$.

We were unable to prove that (III), even together with (I), implies (IV), or to find sequences that satisfy (I) and (III) but do not satisfy (IV).

Question. Does (III) imply (IV)?

All of the above, together with some computational evidence, leads us to the following conjecture on the solution to the invariant factor sum problem:

Conjecture. Given chains $a, b$ and $c$, we have $\mathcal{O}_{c} \subset \mathcal{O}_{a}+\mathcal{O}_{b}$ if and only if (I) holds and there exist units $u$ and $v$ such that $\prod c_{i} \equiv u \prod a_{i}+v \prod b_{i}(\bmod \delta)$, $\prod c_{i} \equiv u \prod a_{i}+(-1)^{n+1} v \prod b_{i}(\bmod \theta)$ and $\prod c_{i} \equiv(-1)^{n+1} u \prod a_{i}+v \prod b_{i}(\bmod \eta)$.

Acknowledgement. We thank the referee for his/her comments. 


\section{Funding}

This work was partially supported by the Centre for Mathematics of the University of Coimbra - UID/MAT/00324/2013, funded by the Portuguese Government through FCT/MEC and co-funded by the European Regional Development Fund through the Partnership Agreement PT2020.

\section{References}

[1] C. Caldeira and J. F. Queiró, Invariant factors of products over elementary divisor domains, Linear Algebra Appl. 485 (2015), 345-358.

[2] W. Crawley-Boevey, On matrices in prescribed conjugacy classes with no common invariant subspace and sum zero, Duke Math. J. 118 (2003), 339352.

[3] S. Friedland, Matrices: Algebra, Analysis and Applications, World Scientific, 2015 .

[4] W. Fulton, Eigenvalues, invariant factors, highest weights, and Schubert calculus, Bulletin AMS 37 (2000), 209-249.

[5] T. Klein, The multiplication of Schur functions and extensions of $p$-modules, J. London Math. Soc. 43 (1968), 280-284.

[6] M. E. Miranda, On the trace of the product and the determinant of the sum of complex matrices with prescribed singular values, in Linear algebra and applications (Vitoria-Gasteiz, 1983), 326-333, Univ. País Vasco-Euskal Herriko Unib., Bilbao, 1984.

[7] J. F. Queiró, Invariant factors as approximation numbers, Linear Algebra Appl. 49 (1983), 131-136.

[8] E. Marques de Sá, Hidden relations for the Smith invariants of a matrix sum, Portugal. Math. 47 (1990), 417-422.

[9] A. P. Santana, J. F. Queiró and E. Marques de Sá, Group representations and matrix spectral problems, Linear and Multilinear Algebra 46 (1999), 1-23.

[10] R. C. Thompson, The Smith invariants of a matrix sum, Proc. AMS 78 (1980), 162-164.

[11] R. C. Thompson, Sums of integral matrices, Linear and Multilinear Algebra 19 (1986), 173-186. 